\title{
A single case study of a family-centred intervention with a young girl with cerebral palsy who is a multimodal communicator
}

\author{
M. B. Wadnerkar, ${ }^{\star}$ T. Pirinen, $\dagger$ R. Haines-Bazrafshan, ${ }^{\star}$ J. Rodgers $\ddagger$ and D. James ${ }^{\star}$ \\ ${ }^{\star}$ NIHR National Biomedical Research Unit in Hearing, University of Nottingham, Nottingham \\ $\dagger$ Ealing and Harrow Community Services, Carmelita House, Ealing, and \\ ¥Institute of Neuroscience, Newcastle University, Newcastle upon Tyne, UK \\ Accepted for publication 19 November 2010
}

\begin{abstract}
Keywords
attunement,

augmentative and

alternative

communication (AAC),

cerebral palsy, eye gaze,

multimodal

communication, video

interaction guidance
\end{abstract}

Correspondence:

Deborah James, NIHR

National Biomedical

Research Unit in Hearing,

Ropewalk House, 113 The

Ropewalk, Nottingham

NG1 5DU, UK

E-mail: deborah.james@

nottingham.ac.uk

\begin{abstract}
Background This paper describes the impact of a family-centred intervention that used video to enhance communication in a young girl with cerebral palsy. This single case study describes how the video-based intervention worked in the context of multimodal communication, which included high-tech augmentative and alternative communication (AAC) device use. This paper includes the family's perspective of the video intervention and they describe the impact of it on their family. Methods This single case study was based on the premise that the video interaction guidance intervention would increase attentiveness between participants during communication. It tests a hypothesis that eye gaze is a fundamental prerequisite for all communicative initiatives, regardless of modality in the child. Multimodality is described as the range of communicative behaviours used by the child and these are coded as AAC communication, vocalizations (intelligible and unintelligible), sign communication, nodding and pointing. Change was analysed over time with multiple testing both pre and post intervention. Data were analysed within INTERACT, a computer software to analyse behaviourally observed data. Behaviours were analysed for frequency and duration, contingency and co-occurrence.

Results Results indicated increased duration of mother's and girl's eye gaze, increased frequency and duration in AAC communication by the girl and significant change in frequency $\left[\chi^{2}(5\right.$, $n=1)=13.25, P<0.05]$ and duration $\left[\chi^{2}(5, n=1)=12.57, P<0.05\right]$ of the girl's multimodal communicative behaviours. Contingency and co-occurrence analysis indicated that mother's eye gaze followed by AAC communication was the most prominent change between the pre- and post-intervention assessments.

Conclusions There was a trend for increased eye gaze in both mum and girl and AAC communication in the girl following the video intervention. The family's perspective concurs with the results.
\end{abstract}

\section{Introduction}

A Cochrane review by Pennington et al. (2003) identified improving communication for both children and parents as the main aim of speech and language therapy for children with cerebral palsy $(\mathrm{CP})$. The review advocates that interventions for CP should be designed to impact on the communicative effectiveness for the child within the context of the family and should include the family's view on the impact of the therapy as part of the evaluation of the intervention's impact. 
The Pennington review (2003) highlighted the importance of changing parental communication pattern to provide more opportunities to children to develop their language and communication skills. The 11 eligible studies in the review (Pennington et al. 2003) indicated that while there was evidence of trends in communication change after therapy, there was very little concrete evidence of positive effects indicating a need to further examine reasons behind the lack of robust evidence from current intervention research about the impact of speech and language therapy for children with CP. The review (Pennington et al. 2003) also highlighted the importance of working on the child's speech production, expressive language skills, comprehension, voice, range of communicative functions and on the use of augmentative and alternative communication (AAC).

Augmentative and alternative communication is an umbrella term that encompasses a variety of methods and technologies designed to compensate for communication limitations of people with spoken language difficulties. AAC can be implemented in unaided forms such as signs or gestures, in low aided forms such as alphabet boards and communication books and in high-tech aided forms such as computer systems to supplement the user's natural communicative range. Implementation of an AAC mode into a child's communicative repertoire aims to overcome barriers with speech production by allowing for a wider range of communicative functions to enable participation and relationship building, reducing challenging behaviours, and serving as a bridge to further language development (Wilkinson \& Hennig 2007).

Success in implementing an AAC system has been quantified by various factors such as frequency and duration of use (Johnson et al. 2006), with greater emphasis been given to user satisfaction, family-centred approach (McNaughton et al. 2008; Iacono \& Cameron 2009), views of communication partners and service providers (Light et al. 2003) and multimodality (Loncke et al. 2006). However, several barriers can get in the way of developing successful AAC communication strategies. These can be inherent to the AAC method itself (Copley \& Ziviani 2004; Bailey et al. 2006; Johnson et al. 2006), or relate to family's misconception about use of AAC replacing spoken communication (Hustad \& Shapley 2003; Wilkinson \& Hennig 2007) making them reluctant to adopt AAC modes (Iacono \& Cameron 2009). Light et al. (1994) describes communicative asymmetry between the AAC user and their communicative partner(s) as another barrier. Behaviours typical of communicative asymmetry can be in terms of imbalanced turn taking, interruptions, reduced initiation by the AAC user, and focus on the technology rather than the individual or the message they are trying to get across (Kent-Walsh \& McNaughton 2005).

Research has suggested that AAC users are likely to assume a passive role in communication when faced with a partner with 'dominating' communicative behaviours (Light et al. 1985a,b; Jolleff et al. 1992). The AAC users' passivity has been interpreted by measurements of aspects such as the range of communicative functions and the diversity of linguistic forms present (Light et al. 1985a,b). One way in which Pennington and McConachie (1999) analysed the issue of communicative role in interaction for children using AAC devices was to define a set of codes to classify structure of conversation, functions of communication and the different modes of communication used between mothers and their children with CP. Pennington and McConachie (1999) coded conversation structure by dividing it into different 'moves': initiations, responses, response/initiations, no response, no response requested and follow-up moves.

In her undergraduate research dissertation, Pirinen (2009) adapted this set of codes in her own evaluation of the impact of a new intervention approach with a young girl who used AAC as part of her multimodal communicative repertoire.

The new intervention approach mentioned in the Pirinen (2009) study is video interaction guidance (VIG). VIG is a therapeutic approach that is underpinned by a premise that communication is a context in which relationships are built. It is principled on getting change by identifying the positive moments of social closeness between the interactants. These positive moments of social closeness are captured on video and the therapist in the VIG approach enables the interactant(s) to microanalyse the communicative elements that led up to a positive moment of social closeness. It focuses on the analysis of attunement and attentiveness between communication partners, is a versatile tool for creating increased awareness of verbal and non-verbal communication, and to promote positive interaction between people. The aim of VIG is to appreciate the strengths in the communication patterns that exist within a dyad and build on them and focuses on change that is important for the family. Successful communication is identified by examining the impact of behaviour on the other person and on the dyad. The theoretical basis for VIG comes from infancy work in developmental psychology (Trevarthen \& Aitken 2001) and from intersubjectivity theory (Trevarthen \& Aitken 2001). The dyadic foundation for VIG means that it focuses on the relational aspects of communication rather than viewing communication as a competency of the individual. See Kennedy and Sked (2008) for a description of the approach and Fukkink (2008) for a meta-analysis of the impact of the intervention in clinical settings. 
In concurrence with Pennington and McConachie's (1999) findings, results from Pirinen's (2009) study demonstrated that the communication partners of the young girl were dominant in the proportions of initiations they made in interactions with her. However, the inherent subjectivity in coding communicative functions such as initiations and responses was a problem because the onset of these communicative acts was very difficult to establish. In addition, the non-verbal communicative behaviours such as eye gaze and nodding were observed to be intensely powerful and intuitively important in the flow of interactions (Pirinen 2009). This level of analysis was not included in the behavioural coding of Pennington and McConachie (1999). So, while Pennington and McConachie (1999) were able to establish good Kappa coefficients for her coding protocol, the validity of the approach was questionable in Pirinen's (2009) application of the coding system. The codes did not appear to represent the details of the highly complex and nuanced communicative behaviours that were unfolding within the interactional dyad between the AAC user and the communicative partners, the user's mother and best friend in the Pirinen study (Pirinen 2009).

As a further aspect of her study, Pirinen (2009) examined the interactions from a more microanalytical perspective with eye gaze as a discrete variable. The importance of eye gaze in communicative function is well established both in normally developing children (Brooks \& Meltzoff 2005) and in children with autism (Loveland \& Landry 1986; Charman et al. 1997). Co-ordinated attention is mediated through eye gaze and is highly relevant in meaningful communication (Carpenter et al. 1998; Charman et al. 2000; Legerstee et al. 2007). Eye gaze as a measure of social communicative function is both theoretically and methodologically robust. Data from the evaluation by Pirinen (2009) showed that eye gaze between the interactants was significantly longer in duration as a result of a new intervention and this was found across multiple baseline measures and post-intervention measures in two dyadic contexts.

Pirinen (2009) coded eye gaze traditionally using a stop watch and video recording and this method of coding eye gaze could possibility be less precise because of methodological restrictions. In the current paper we set out to measure eye gaze with more precision and to build on the results of the previous work to assess whether enhanced eye gaze was related to enhanced communicative function in the girl who used AAC as part of her multimodal communicative repertoire. We set out to test the hypothesis that eye gaze is a critical factor in communicative function by looking at the contingency of eye gaze behaviour on communicative functions in both members of the dyad the mother and the girl. In the current study, enhanced communicative function will be explored by looking at increased duration and frequency of a range of communicative acts (speech, sign, gesture, nodding, pointing and AAC use). In addition to the application of microanalytical approach to the measurement of behaviour, we also set out to include the users' views of the impact of the intervention and the validity of the results as interpreted in this paper based on their own perspective (see Box 1). As per our knowledge, this is the first study where the approach has been applied in the context of children with specific language and communication needs.

\section{Methods}

This paper extends findings from the Pirinen (2009) dissertation and uses a new methodology to code and analyse the behavioural data. Information on participants and testing procedure comes from Pirinen's (2009) dissertation.

\section{Participants}

The main participant was an 11-year-old girl, referred to as $\mathrm{B}$ in this paper. B has athetoid CP affecting all four limbs. She lacks muscle co-ordination required for walking independently with ease and for clear, intelligible speech. She can drive a power wheelchair and walk when aided and has recently started walking short distances on her own. She uses a multimodal approach to communication, which includes: a high-tech communication aid (a Dynavox), some signs, gestures, verbal attempts and vocalizations. Her verbal communication and signs are intelligible mainly to communication partners familiar to her and they often require repetition or modification for clarification purpose. She has age appropriate receptive and cognitive skills and currently attends a special needs school for student's with physical disability. B has previously been included in a mainstream school on a part-time basis, and is currently considering reintegration into a mainstream setting on a part-time basis. B is a social person, who is keen to communicate with family, friends and peers. B's parents and best friend (referred to as R) also participated in the study.

B comes from a monolingual English speaking family. At the time of the original study, B's mother and father were both in employment with professional job roles.

Pirinen gained ethical approval from the Speech Language Sciences Section Research Ethics Committee of Newcastle University. Parental and child consent was gained for the intervention and for the study. Consent was also taken and granted for continued use of the data by the group lead and one of the authors D. James. 


\section{Box 1. Parent and girl perspective.}

\section{Parent perspective}

The approach

'The emphasis from the services we have received for so much of B's life have been about identifying and trying to address things that B cannot do as well as she (and we!) would like. Obviously the ability to communicate using her voice has been a major focus of the services we have received. The terms communication impairment, language impaired, etc. have been and still are used frequently and clearly cast B's communicative attempts in a negative and "sub-standard" light. This medicalization has had a significant impact on all of us. Participating in the intervention has really changed our views of B's capacity to communicate. Using the VIG techniques to watch the very sophisticated and subtle methods that B uses to get her message across has made us realize that $\mathrm{B}$ is a expert and incredibly skilled communicator, who despite the limited tools available to her is participating as an effective communicator in a complex social world'.

The findings

'The findings mirror the positive impact that we feel the intervention has had on our family. B uses the methods within her repertoire (AAC, signs, gesture, facial expression, verbalization) increasingly more confidently and effectively and seems increasingly skilled in making judgements about the readiness and capability of the listener to be able to access her communication attempts and switch into a different mode as and when needed. While this could simply be a product of developmental progression it could also be suggested that participating in VIG allowed B to explore the reactions and responses of the listener during an interaction in a way that would not be feasible in real time and that she is now more attuned to what to look out for. Feedback from school would indicate this increasingly flexibility is apparent in that setting too. An important aspect of the intervention for us as a family was the emphasis on the cooperative nature of the interactional process and that effective communication takes place in the space in between the communicator and the listener. This clearly emphasized that we all have an important role in the transaction and that the best communication occurs when all are actively engaged in the process'.

\section{B's perspective}

'I liked the study. It was good to watch the videos and talk about my talking'.

\section{Testing procedure}

This single case study used a repeated design to measure changes pre and post intervention.

\section{Pre-intervention stage}

In the original study two sets of dyadic interactions were measured: interactions between $B$ and her mother, and B and her best friend R. Before VIG intervention, four baseline measures focussing on these dyads were attained. B was filmed interacting in both dyads for $20 \mathrm{~min}$ each, weekly for 4 weeks. Filming of the dyads took place in B's home, with the aim to capture video footage of B interacting in her natural environment. During the dyadic interactions, participants had access to a range of books, games and magazines typically of interest to B, promoting a natural environment for interactions. In the majority of video clips, participants selected one stimulus on their own, for example, a book or magazine as the main object of shared interest, which served to initiate further topics of conversation. Filming was done by a familiar person (Pirinen) in a naturalistic setting and the camera was placed in position at the start of the session.

See proceeding Coding procedure section for details of new analysis of video data.

\section{Intervention stage}

Four sessions of VIG intervention were carried out. This has been found to be the optimal frequency of VIG sessions to attain most effective outcomes (Bakermans-Kranenburg et al. 2003; Fukkink 2008). A trained VIG practitioner/guider (D. James) carried out the intervention following the structured protocol of the VIG procedure. As the VIG intervention progressed, B's mother, father and $\mathrm{R}$ all became part of the intervention process. The dynamic involvement of these various communication partners was not prescribed by the VIG guider, but rather led by the participants and their wishes. The intervention 
consisted of four films taken at home with B interacting with different participants and four shared review sessions, which were scheduled to take place about a week after the films were recorded. At the shared review sessions, three short edited clips were replayed for the participants to microanalyse and use as a basis of shared understanding of the reasons why that communicative moment was successful. For more information on the nature of the intervention at a generic level, see Kennedy and Sked (2008). Further detail on the way the participation happened during the intervention in this case is provided below.

- Four films of around 10-20 min in duration were taken of B interacting with (a) her mother, (b) her best friend, (c) her father, (d) her mother and her mother and father in her home (in this chronological sequence).

- These films were recorded by (a) the intervention guider, (b) her mother, (c) and (d) - her parents.

- All films were analysed for micro moments of successful interaction by the intervention guider, and each of B's parents analysed the films of themselves interacting with B (films c and d) and they each selected one micro moment of successful interaction to share with each other and the interaction guider during the final shared review session.

- The first shared review session was with the intervention guider and B's mother; the second shared review session was with $\mathrm{B}$, her best friend, her mother and the guider; the final two shared review sessions were with the intervention guider, the mother and the father. B's mother was able to facilitate an additional review session where the participants were $B$ and her best friend and this took place between shared reviews 2 and 3 .

\section{Post-intervention stage}

Once the VIG intervention was delivered, post-intervention baseline measures were assessed. As in the pre-intervention stage, both the mother and B were filmed interacting for 20 min on four occasions weekly. In order to triangulate the quantitative analysis, post-intervention unstructured interviews were carried out with B's parents, to find out their views on the effect of the intervention. The family were blind to the variables to be coded in the analysis of the impact of the intervention. Views from B's mother and B on the impact of the intervention on them and their personal reflections on the results reported in this paper were gathered and are presented in Box 1.

\section{Coding procedure}

The original video recordings were analysed in INTERACT (INTERACT 2008). INTERACT allows precise coding of behaviour from audio-video footage. It can also perform pattern extraction to identify frequency of sequences of behaviour between interactants. Pirinen (2009) used altogether eight video segments, four in the pre-intervention and four in the postintervention stage. For the purpose of this new analysis two video segments from pre intervention and two from post intervention were chosen randomly and were combined as one video segment each for pre intervention and post intervention. This was done so that the new microanalytical behavioural coding could be well managed in time. Overall duration of the preintervention video footage was $10 \mathrm{~min} 29 \mathrm{~s}$ and for post intervention was $10 \mathrm{~min} 59 \mathrm{~s}$. Total duration of footage analysed was $5 \mathrm{~min} 46 \mathrm{~s}$ at pre intervention and $6 \mathrm{~min} 23 \mathrm{~s}$ at post intervention. Video footage was observed in a frame-by-frame manner to mark the exact duration of each event (h00:min00:s00:f00). For enhanced accuracy, the vocalizations were first coded separately in Praat (Boersma \& Weenink 2010) from waveforms with the durations later entered onto INTERACT. Each video segment is referred to as a dataset in the analysis and results section.

In this study we take two main measures of the intervention success. We elicited the family's views of the intervention and we coded eye gaze in both the mother and girl who took part in the study. In our secondary question we wanted to find out if eye gaze was a mechanism that was related to enhanced communicative repertoire in the child. Thus eye gaze in this context is described as an explanatory variable and the response variables or multimodal communicative behaviours were AAC communication, intelligible vocalizations, unintelligible vocalizations, sign communication, nodding, pointing and girl looking at mum. A detailed coding system was developed towards this end for all the behavioural codes. See Appendix I for explanation of each code and how it was coded.

The first author independently coded $20 \%$ of the video and audio for inter-rater agreement for both pre and post stages for Girl and Mother. There was a good agreement between the first and the second coder as indicated by the Kappa coefficient (Cohen's Kappa), of $\kappa=0.83$ for girl and mother.

\section{Data analysis}

Data were analysed within INTERACT (INTERACT 2008) using the analysis tool. Data are presented separately for eye gaze and the response variables pre and post intervention. 
In the first instance, duration and frequency count of behavioural codes is described. Co-occurrence of codes was also computed from timeline chart within INTERACT. The entire duration of pre-intervention ( $5 \mathrm{~min} 46 \mathrm{~s}$ ) and postintervention (6 min $23 \mathrm{~s})$ session was taken in the timeline. A chi-squared test of goodness-of-fit was performed to determine whether there was a significant difference between the duration and frequency changes seen at post intervention. Co-occurrence was calculated to find simultaneously occurring pairs of behaviours, which overlapped in time within each dataset.

Contingency analysis was performed to further understand how close together behaviours that follow each other within a predefined time frame are. As behavioural contingency is the main proposition of this study mum looking at girl was compared with all behaviours for the girl. This was done to further understand which behaviours drive the pattern seen in the co-occurrence and lag sequential analysis results. Time period for contingency analysis was taken as $0 \mathrm{~s}$ to get a pure count of closely occurring behaviours.

\section{Results}

Results indicated a change in behaviour patterns pre and post intervention. However, the change was bidirectional with some behaviour showing an increase and some behaviour showing a decrease in the count and duration following intervention.

Frequency count and duration: change over time.

\section{Eye gaze}

Post-intervention frequency count of the number of occasions of girl looking at mum remained almost same (pre-intervention count: 40 and post-intervention count: 41 ), whereas greater instances were seen of mum looking at girl (pre-intervention count: 50 and post-intervention count: 58). However, a highly noticeable increase in duration was observed indicating greater degree of perseverance for eye gaze following intervention on the girl (duration pre intervention: $170.88 \mathrm{~s}$ and duration post intervention: $326.04 \mathrm{~s}$ ) and on the mother's part (duration pre intervention: $277.48 \mathrm{~s}$ and duration post intervention: $384.96 \mathrm{~s}$ ) (Fig. 1).

\section{Response variables}

Post-intervention frequency count indicated an increase in AAC communication (pre-intervention count: 5 and postintervention count: 22) and nodding (pre-intervention count: 25 and post-intervention count: 43). Sign communication

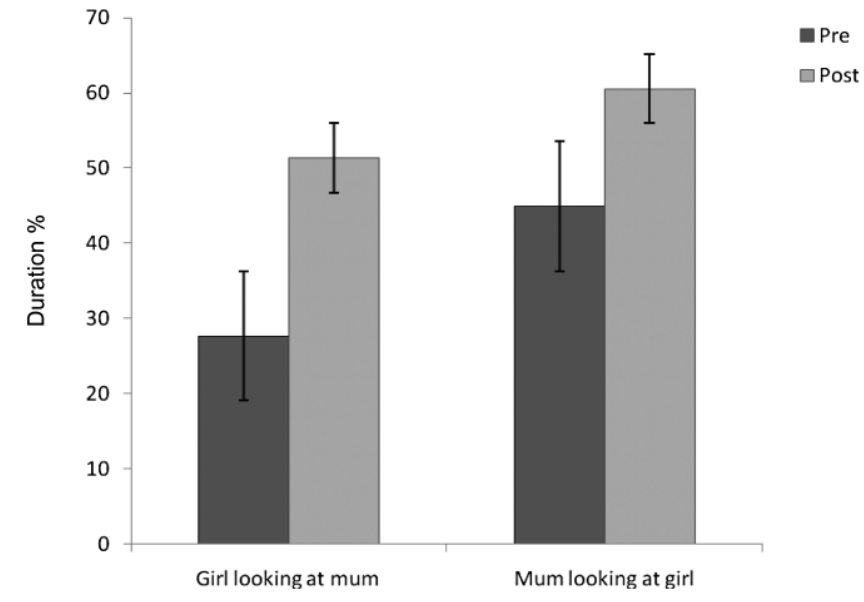

Figure 1. Duration (\%) pre and post intervention for eye gaze.

(pre-intervention count: 5 and post-intervention count: 12) also increased and pointing showed only a slight increase (pre-intervention count: 15 and post-intervention count: 17). A decrease was seen in unintelligible vocalizations (preintervention count: 47 and post-intervention count: 24) and intelligible communication (pre-intervention count: 23 and post-intervention count: 12; Fig. 2).

Changes in duration indicated that following intervention $\mathrm{B}$ was persevering more with using AAC communication (duration pre intervention: $3.48 \mathrm{~s}$ and duration post intervention: $12.2 \mathrm{~s}$ ). She showed decreased perseverance for unintelligible vocalizations (duration pre intervention: $58.64 \mathrm{~s}$ and duration post intervention: $29.32 \mathrm{~s}$ ). Nodding and pointing showed decreased perseverance following intervention (Fig. 3).

Chi-squared test indicated a significant change at post intervention in the frequency $\left[\chi^{2}(5, n=1)=13.25, P<0.05\right]$ and the duration $\left[\chi^{2}(5, n=1)=12.57, P<0.05\right]$ of response variables.

\section{Contingency analysis}

This trend was further explored through contingency analysis. With no time gap between behaviours (time frame of $0 \mathrm{~s}$ ) contingency of mum looking at girl followed by AAC communication was seen to be increased from 0 at pre intervention to 2 at post intervention (this remained at 2 within a time frame of $1 \mathrm{~s}$ and increased to 3 within a time frame of $5 \mathrm{~s}$ in post intervention). Rest of the pairs did not show any noteworthy contingent patterns (Table 1). Thus an analysis of contingency was useful in identifying the behaviours that had a more stable and consistent pattern of co-occurring change. 
Figure 2. Frequency of codes for girl's multimodal communicative behaviours at pre and post intervention. AAC, augmentative and alternative communication

Figure 3. Duration (\%) pre and post intervention for girl's multimodal communicative behaviours. AAC, augmentative and alternative communication.
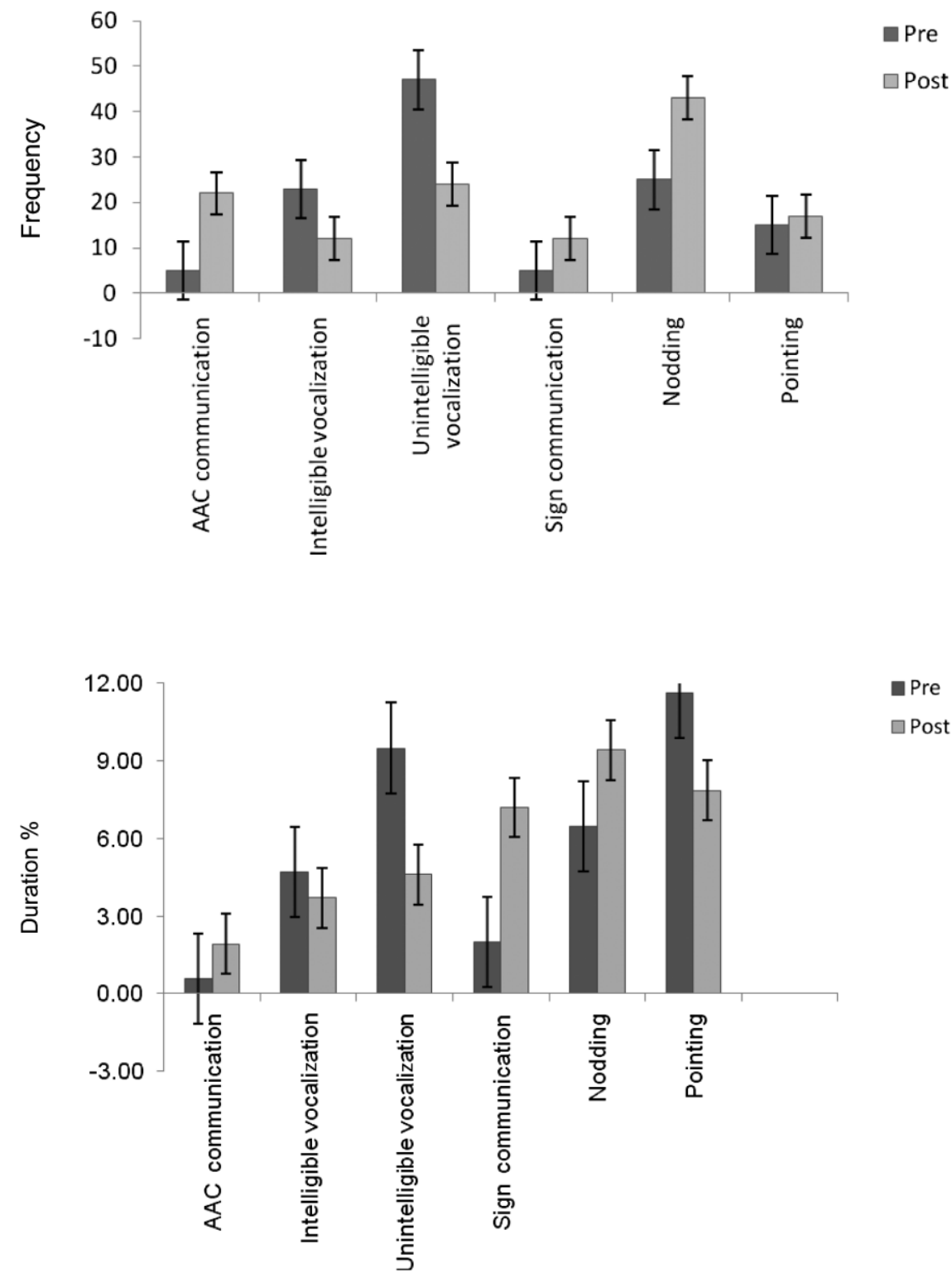

Table 1. Contingency analysis within a time period of $0 \mathrm{~s}$ for mother's eye gaze and girl's multimodal communicative behaviours

\begin{tabular}{lll}
\hline & \multicolumn{2}{l}{ Mum looking at girl followed by codes for girl } \\
\cline { 2 - 3 } Code & Pre intervention & Post intervention \\
\hline AAC communication & $0^{*}$ & $2^{*}$ \\
Looking at mum & 0 & 1 \\
Intelligible vocalization & 0 & 0 \\
Unintelligible vocalization & 0 & 0 \\
Sign communication & 0 & 0 \\
Nodding & 0 & 0 \\
Pointing & 0 & 0 \\
\hline
\end{tabular}

*These remained at 2 within a time frame of $1 \mathrm{~s}$ and increased to 3 within a time frame of $5 \mathrm{~s}$ in post intervention.

$A A C$, augmentative and alternative communication. 


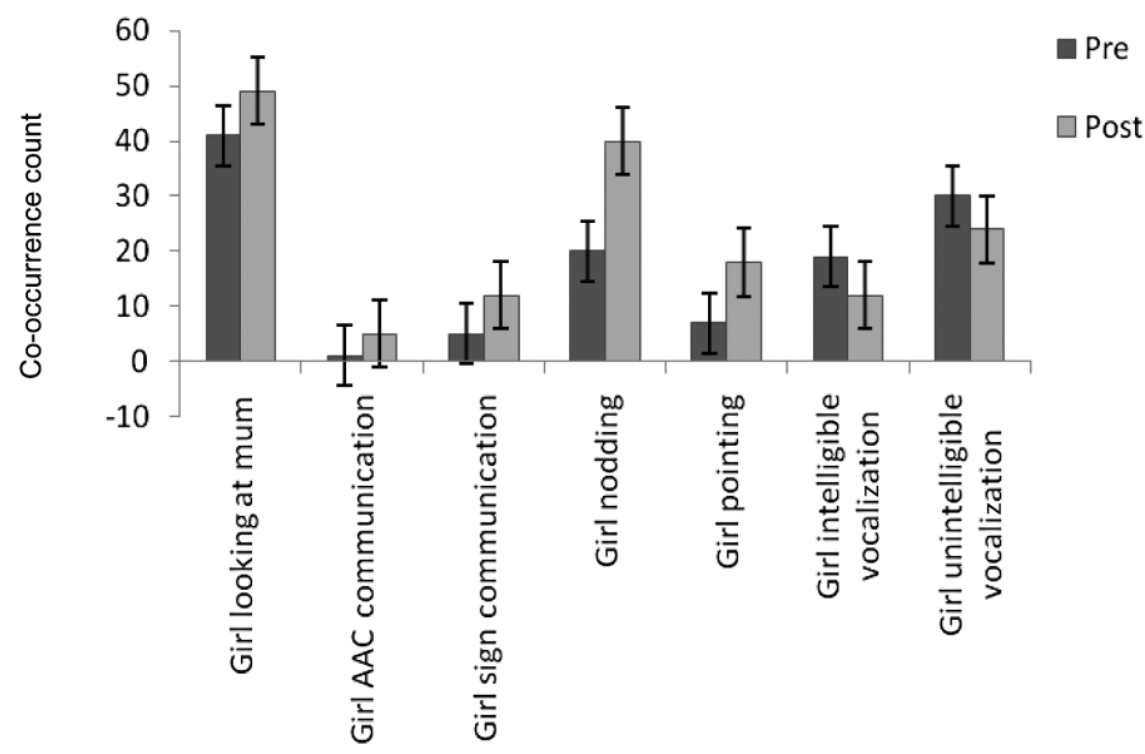

Figure 4. Co-occurrence of mum looking at girl with girl's multimodal communicative behaviours at pre and post intervention.

\section{Co-occurrence}

Figure 4 shows results for the co-occurrence of behavioural codes. Each code in girl was paired with mum looking at girl using the co-occurrence filter. Results indicated an interesting pattern pre and post intervention. AAC communication (increased by four counts), girl looking at mum (increased by eight counts), nodding (increased by 20 counts), pointing (increased by 11 counts) and sign communication (increased by 7 counts) all showed an increased co-occurrence with mum looking at girl at post intervention. Both intelligible and unintelligible vocalizations showed a reduced co-occurrence at post intervention (decrease by seven and six counts, respectively; Fig. 4).

To summarize, at post intervention an increase in eye gaze was seen to be independently co-occurring with increase in AAC communication, decrease in unintelligible vocalizations. Overall, there seemed to be a trend for increase in eye gaze in both mum and B (mum looking at girl and girl looking at mum) and AAC communication in girl following the intervention. Views and reflections from B's mother and B also concur with the findings (see Box 1).

\section{Discussion}

Cerebral palsy is a heterogeneous condition and interventions for CP reflect this heterogeneity. Children with $\mathrm{CP}$ can have a difficulty in acquiring age appropriate speech and language skills and show reduced functional communication skills (Jolleff et al. 1992). Difficulties can be experienced in initiation (Pennington
\& McConachie 1999), turn taking, repair and maintaining the flow of conversation (Light et al. 1985a,b). The family context in which these variations arise either because of the condition or the intervention add another dimension to the complexity. Traditionally, speech and language therapy interventions follow the medical model where the target of the intervention is the impairment and the result of intervention is expected to generalize into the real world communicative contexts of the child. In the VIG intervention rather than intervening to change the impairment, the focus is to work the communicative strengths that exist within the relationships that the family believe are meaningful to them and their child. Thus, in this approach the family and the social context in which the child/family operate are the basis of the intervention. In this case study the family had experienced different intervention approaches over the years and they found this family-centred approach particularly helpful. It gave them new insights into their daughter's communicative strengths and new way of thinking about communication in general, 'an important aspect of the intervention for us as a family was the emphasis on the cooperative nature of the interactional process and that effective communication takes place in the space in between the communicator and the listeners'. The results from this case study are in line with the calls from the field for more user-centred (Iacono \& Cameron 2009) and family-centred (Pennington et al. 2003) approaches.

There is a fair amount of published work on the importance of eye gaze, its role in shared attention and co-ordinated attention in the fields of normal development (Brooks \& Meltzoff 2005) and autism (Loveland \& Landry 1986; Charman et al. 1997). However, when it comes to the case of multimodal 
communication, there has typically been more of a focus on the technology of the device, and the motor acts of signing, gesturing, nodding and pointing. Our results suggest that eye gaze is a critical component in enhancements in multimodal communicative acts. Given the multimodal nature of communication in children with CP who are AAC users, there is a need to explore further the role of attention, with eye gaze as a critical component of that, for this group of communicators.

As outlined in the Introduction and Methods sections, there are many challenges in getting valid and reliable coding of communicative behaviour. In this study, we were able to adopt a microanalytical approach to the behavioural coding and we found it easy to gain inter-rater reliability for behaviour and duration of eye gaze and the motor acts (nodding, pointing, signing and use of AAC); however, what was more difficult was the classification of the status of B's vocal utterances. It was somewhat subjective to classify utterances as either intelligible or non-intelligible and that is because we relied on the response of the interactant to decide whether B's utterance had been understood by the listener or not. This means that we are less sure about the interpretation of the changes in vocal utterances. It is indicative of the difficulties of behavioural coding of acts, such as talking, as these could almost be described as being the properties of the communicative space between the interactants (see mother's comments in Box 1).

This brings us to a final consideration of the underlying theoretical premise in which we work. In much of speech and language therapy the underlying assumption is that communication is the property of an individual and that communication is an act of sending and receiving messages. In many ways, the methodological challenge of coding speech/language for individuals has indicated the flaws inherent in this basic assumption. Rather than seeing communication as a send/receive act of transmission, we can also understand communication at a more fundamental level - it is the central energy that binds humans into relationships of trust and security. Focusing on the relational properties of communication will allow us to rethink how we should design interventions for those who, for whatever reason, face challenges in communicating.

\section{Key messages}

- A family-centred intervention approach that focused on successful communication was considered by the family to be beneficial in developing their understanding of communication within the family. Their own subjective perception of change that they attributed to the intervention aligned with the results reported in this paper.

- The most striking finding in this case study was that of a large increase in the use of the high-tech AAC device at the post-intervention stage. In this study we found a contingency between AAC use and eye gaze between the interactants.

- Rather than making the technology the focus of communicative therapy for children who use high-tech communication aids, we suggest that focusing on communication in its most fundamental function, that of, building relationships has potential for significant generalized impact for multimodal communicators.

- Further research on the importance of shared attention and attunement in multimodal communication could yield significant advances in our understanding of the properties of successful therapeutic interventions.

\section{Acknowledgements}

The NIHR National Biomedical Research Unit in Hearing (DJ, MW and RHB) is funded by the National Institute for Health Research.

\section{References}

Bailey, R. L., Parette, H. P. Jr, Stoner, J. B., Angell, M. E. \& Carroll, K. (2006) Family members' perceptions of augmentative and alternative communication device use. Language, Speech, and Hearing Services in Schools, 37, 50-60.

Bakermans-Kranenburg, M. J., van Ijzendoorn, M. H. \& Juffer, F. (2003) Less is more: meta-analyses of sensitivity and attachment interventions in early childhood. Psychological Bulletin, 129, 195-215.

Boersma, P. \& Weenink, D. (2010) Praat: doing phonetics by computer [computer program] (Version 5.1.30). Available at: http://www.praat.org/ (accessed from 20 March 2010).

Brooks, R. \& Meltzoff, A. N. (2005) The development of gaze following and its relation to language. Developmental Science, 8 , 535-543.

Carpenter, M., Nagell, K. \& Tomasello, M. (1998) Social cognition, joint attention, and communicative competence from 9 to 15 months of age. Monographs of the Society for Research in Child Development, 63, 1-143.

Charman, T., Swettenham, J., Baron-Cohen, S., Cox, A., Baird, G. \& Drew, A. (1997) Infants with autism: an investigation of empathy, pretend play, joint attention, and imitation. Developmental Psychology, 33, 781-789. 
Charman, T., Baron-Cohen, S., Swettenham, J., Baird, G., Cox, A. \& Drew, A. (2000) Testing joint attention, imitation, and play as infancy precursors to language and theory of mind. Cognitive Development, 15, 481-498.

Copley, J. \& Ziviani, J. (2004) Barriers to the use of assistive technology for children with multiple disabilities. Occupational Therapy International, 11, 229-243.

Fukkink, R. (2008) Video feedback in widescreen: a meta-analysis of family programs. Clinical Psychology Review, 28, 904-916.

Hustad, K. \& Shapley, K. (2003) AAC and natural speech in individuals with developmental disabilities. In: Communicative Competence for Individuals Who Use AAC: From Research to Effective Practice (eds J. C. Light, D. R. Beukelman \& J. Reichle), pp. 41-62. Paul H. Brookes Publishing Company, Baltimore, MD, USA.

Iacono, T. \& Cameron, M. (2009) Australian speech-language pathologists' perceptions and experiences of augmentative and alternative communication in early childhood intervention. Augmentative and Alternative Communication, 25, 236-249.

INTERACT (2008) (Version V1.3): mangold International.

Johnson, J. M., Inglebret, E., Jones, C. \& Ray, J. (2006) Perspectives of speech language pathologists regarding success versus abandonment of AAC. Augmentative and Alternative Communication, 22, 85-99.

Jolleff, N., McConachie, H., Winyard, S., Jones, S., Wisbeach, A. \& Clayton, C. (1992) Communication aids for children: procedures and problems. Developmental Medicine and Child Neurology, 8, 719-730.

Kennedy, H. \& Sked, H. (2008) Video interaction guidance: a bridge to better interactions for individuals with communication impairments. In: Promoting Social Interaction for Individuals with Communicative Impairments: Making Contact (ed. M. S. Zeedyk), pp. 139-154. Jessica Kingsley Publishers, London, UK.

Kent-Walsh, J. \& McNaughton, D. (2005) Communication partner instruction in AAC: present practices and future directions. Augmentative and Alternative Communication, 21, 195-204.

Legerstee, M., Markova, G. \& Fisher, T. (2007) The role of maternal affect attunement in dyadic and triadic communication. Infant Behavior \& Development, 30, 296-306.

Light, J., Collier, B. \& Parnes, P. (1985a) Interaction between young non-speaking physically disabled children and their primary caregivers. Part 2: communicative function. Augmentative and Alternative Cornniunication, 1, 98-107.

Light, J., Collier, B. \& Parnes, P. (1985b) Interaction between young non-speaking physically disabled children and their primary caregivers. Part 1 : discourse patterns. Augmentative and Alternative Communication, 1, 74-83.

Light, J., Binger, C. \& Smith, A. K. (1994) Story reading interactions between preschoolers who use AAC and their mothers. Augmentative and Alternative Communication, 10, 255-268.

Light, J., Beukelman, D. R. \& Reichle, J. (2003) Communicative Competence for Individuals Who Use AAC: From Research to Effective Practice. Brookes Publishing Co., Inc., Baltimore, MD, USA.

Loncke, F. T., Campbell, J., England, A. M. \& Haley, T. (2006) Multimodality: a basis for augmentative and alternative communication psycholinguistic, cognitive, and clinical/ educational aspects. Disability and Rehabilitation, 28, 169-174.

Loveland, K. A. \& Landry, S. (1986) Joint attention and language in autism and developmental language delay. Journal of Autism and Developmental Disorders, 16, 335-349.

McNaughton, D., Rackensperger, T., Benedek-Wood, E., Krezman, C., Williams, M. B. \& Light, J. (2008) 'A child needs to be given a chance to succeed': parents of individuals who use AAC describe the benefits and challenges of learning AAC technologies. Augmentative and Alternative Communication, 24, 43-55.

Pennington, L. \& McConachie, H. (1999) Mother-child interaction revisited: communication with non-speaking physically disabled children. International Journal of Language \& Communication Disorders, 34, 391-416.

Pennington, L., Goldbart, J. \& Marshall, J. (2003) Speech and language therapy to improve the communication skills of children with cerebral palsy. Cochrane Database of Systematic Reviews, Issue 2, Art. No.: CD003466.

Pirinen, T. (2009) A single case study evaluating the impact of video-interaction-guidance: empowering a young girl who has cerebral palsy and uses multi-modal communication as a communicator. Unpublished BSc (Hons) Speech and Language Sciences Dissertation. Newcastle University, Newcastle, UK.

Trevarthen, C. \& Aitken, K. J. (2001) Infant intersubjectivity: research, theory and clinical applications. Journal of Child Psychology and Psychiatry, and Allied Disciplines, 42, 3-48.

Wilkinson, K. M. \& Hennig, S. (2007) The state of research and practice in augmentative and alternative communication for children with developmental/intellectual disabilities. Mental Retardation and Developmental Disabilities Research Reviews, 13, 58-69. 


\section{Appendix I}

Behavioural coding scheme

\begin{tabular}{|c|c|c|c|c|}
\hline Code & Class & Code description & Start code & Last frame coded \\
\hline AAC Communication & Girl & $\begin{array}{l}\text { Voice of Dynavox (not coded if it was } \\
\text { a button B accidentally pushed as } \\
\text { she was trying to find the word she } \\
\text { wanted) }\end{array}$ & $\begin{array}{l}\text { Beginning of Dynavox voice on } \\
\text { waveform }\end{array}$ & End of Dynavox voice on waveform \\
\hline Girl looking at mum & Girl & $\begin{array}{l}\text { Coded when girl's eyes are visible and } \\
\text { they are looking at the face of the } \\
\text { mother }\end{array}$ & $\begin{array}{l}\text { When eyes first come in line with } \\
\text { mother's face }\end{array}$ & $\begin{array}{l}\text { Frame before eyes first turn away } \\
\text { from mother's face }\end{array}$ \\
\hline Intelligible vocalization & Girl & $\begin{array}{l}\text { Interlocutor lexically understood } \\
\text { vocalization }\end{array}$ & $\begin{array}{l}\text { Beginning of vocalization on } \\
\text { waveform }\end{array}$ & End of vocalization on waveform \\
\hline Unintelligible vocalization & Girl & $\begin{array}{l}\text { Interlocutor did not understand or if } \\
\text { there was no probably lexical } \\
\text { content to what was said }\end{array}$ & $\begin{array}{l}\text { Beginning of vocalization on } \\
\text { waveform }\end{array}$ & End of vocalization on waveform \\
\hline Sign communication & Girl & Intelligible signing or gesture & $\begin{array}{l}\text { Start of arm movement which } \\
\text { becomes sign }\end{array}$ & $\begin{array}{l}\text { Frame right before hand first } \\
\text { 'releases' the sign }\end{array}$ \\
\hline Girl nodding & Girl & Nodding & First head movement of the nod & $\begin{array}{l}\text { Frame before head comes to a still } \\
\text { point at end of nod }\end{array}$ \\
\hline Girl pointing & Girl & B pointing at an object & $\begin{array}{l}\text { Start of arm movement which } \\
\text { becomes a point }\end{array}$ & $\begin{array}{l}\text { Frame right before pointing finger } \\
\text { starts to 'unform' the point }\end{array}$ \\
\hline Mum looking at girl & Mother & $\begin{array}{l}\text { Coded when mother's eyes are visible } \\
\text { and they are looking at girl's face }\end{array}$ & $\begin{array}{l}\text { When angle of eyes first is in direction } \\
\text { of girl's face }\end{array}$ & $\begin{array}{l}\text { Frame before eyes first turn away } \\
\text { from girl's face }\end{array}$ \\
\hline
\end{tabular}

